\title{
Data Analytic Techniques for Developing Decision Support System on Agrometeorological Parameters for Farmers
}

\author{
Sowmya B.J., M. S. Ramaiah Institute of Technology, Bengaluru, India \\ Krishna Chaitanya S., M.S. Ramaiah Institute of Technology, Bengaluru, India \\ Seema S., M.S. Ramaiah Institute of Technology, Bengaluru, India \\ K.G. Srinivasa, National Institute of Technical Teachers Training and Research, Chandigarh, India \\ https://orcid.org/0000-0003-1022-8431
}

\begin{abstract}
The day-to-day lives of humans are changing remarkably due to the evolution in tools, techniques and technology across the planet. This evolution is not only impacting the growth of humans but also contributing to the growth and well-being of society and country. The domain of data analytics (DA) and internet of things (IoT) is very much facilitating this growth. But there have been only a handful of innovations and explorations in the field of agriculture, although it being the backbone and largely contributing to the gross domestic product (GDP) of a country like India. The reason for it may be profuse, such as the erratic weather conditions, improper irrigation, farmers being skeptical using modern tools and many more. But being in a developing country that has its primary focus on invention and innovation, a consensus has to be reached so that the modern tools and technologies, abet agriculture throughout the country. In our work, an attempt is made to analyze the different aspects that influences the variable outcomes in agriculture with the aid of various data analytic algorithms. Rainfall, humidity and temperature are some of the variables that determine the type of crop. Therefore, the task of prediction of crop type given these factors using decision trees and support vector machines (SVM) is implemented, and the accuracy of the models are computed. Here, more focus is given to the state of Karnataka and to its major crops. With rice, ragi and maize being some of the predominant crops, an analysis is portrayed considering the yield across the state.
\end{abstract}

\section{KEYWORDS}

Classification and Regression Trees (CART), Crop Type Prediction, Decision Support System, Smart Agriculture, Support Vector Machines (SVM) 


\section{INTRODUCTION}

The advancements and improvements happening in technologies have fueled the data being generated to escalate rapidly, making it excruciatingly difficult to be handled by traditional databases and thus embarking upon the age of Big Data. The field of artificial intelligence (AI), Cloud computing, Internet of Things (IoT) among other things are aiding the development of Big data to a great extent. IoT aspires to combine the real world with the virtual world by making use of the Internet as intermediary to communicate and exchange information without the requirement of any human interaction (Elijah, Rahman et al., 2018). An analogous to IoT is the cyber physical system (CPS) that combines the capabilities of physical components with computing and communicational components.

IoT is concerned with the acquisition, storing and processing of data and does not provide many computational capabilities and that is where the field of data analytics (DA) plays a pivotal role. Based on the need of IoT applications, DA is further categorized into different types such as memory-level analytics, real-time analytics, off-line analytics, massive analytics and business intelligence level analytics. One of the application domains in CPS is smart farming or smart agriculture - which is an approach of administering the farming with the use of present-day information and communication technologies (ICT) to enhance the quality and quantity of yield. The technologies include IoT-based hardware and software, communication technologies and data analytic techniques that assist in decision making process (sciforce). Image processing is one of the DA techniques that is used extensively in agriculture as mostly the data acquired through IoT consists of images, audio, and video. The combination of Data analytics with IoT is proving to be a force as it is boosting to accomplish higher yield production and crop failure reduction.

But even with these ever-changing technologies, the amount of innovations and explorations in the field of Agriculture is only few. In a country like India, Agriculture is much more than a feeding source, as it is the major source of food production and contributes largely to the nation's gross domestic product (GDP). There has been a slump in GDP of India in the past few years with respect to agriculture due to factors such as irregular weather conditions resulting in crop damage and inexperience of using modern technologies. Fresh water is also not being properly utilized for irrigation. Due to the erratic climatic conditions, improper irrigation and inexperience of use of modern technologies and pesticides has led to the damage of crops, increase in plant diseases and thereby threatening food security. Crop diseases contribute directly and indirectly to the spread of human infectious diseases and environmental damage. Hence, this work intends to provide a decision support system based on the agrometeorological conditions that directly or indirectly affects the growth of crops.

\section{RELATED WORK}

Before diving into the actual implementation of the Agricultural decision support system, there are some noteworthy related works that have already made an impact around the globe in the field of agriculture. Some of them are pictured below that uses different data analytic techniques to solve the existing problems.

The rudimentary task of an agriculture decision support system would be the crop type and yield prediction. Sujatha and Isakki in their work make use of different classification algorithms such as Naïve Bayes and Decision tree for forecasting the crop yield (Sujatha and Isakki, 2016). The system proposed by them contains an input module (comprising of crop name, land area, soil type, soil $\mathrm{pH}$, pest details, weather, water level, seed type) and feature selection unit (minimizes the selection set of an attribute based on crop details). Eventually, after feature selection the data is grouped with similar contents on the basis of defined classification rules, which are then implemented to categorize the crop depending on the name, yield and pesticide.

Similarly, Vijayabaskara et al. (2017) in their work use predictive analytics (numerous statistical techniques to interpret historic and present data and forecast based on it) to predict the crop. An 
application to test the soil fertility, suggest or predict the crop and its yields, and recommendations related to fertilizers is provided and as the prediction depends on the atmosphere which is not constant, the results may vary accordingly.

As noted above, there are many factors on which a crop type and yield relies upon. Afrin et al. (2018) predict the crop yield of Bangladesh based on the soil and climate factors. In their work, comparison of four different clustering (K-Means, Partition among Medoids (PAM), CLARA and DBSCAN) and four different regression (simple, multiple, step-wise and generalized) algorithms are made, finally concluding that PAM gives better results compared to other clustering techniques and generalized linear model has lowest Root Mean Square Error (RMSE) compared to other regression techniques and suggest the same for forecasting the crop yield.

On the similar lines, Pudumalar et. al., in their proposal portray an ensembling technique that uses Majority Voting technique to predict the crops that combines the competency of numerous models to achieve better efficiency and accuracy than a single model considered alone (Pudumalar et al., 2016). The voting technique uses chi-squared automatic interaction detection (CHAID), K-nearest neighbour, random tree and Naïve-Bayes as the base learner models. The work focuses on the soil specific attributes that could act as the deciding factors in the prediction of type of crops. They develop a crop recommendation system based on the ensemble technique and assert that the accuracy of crop type predictions accounts to $88 \%$.

Also noting that the data in Agriculture is very vast that there are many factors like the type of soil, $\mathrm{pH}$ level of the soil, temperature, longitude, latitude, monthly rainfall and many more affecting a small process and hence any decision to be taken should be more accurate. Doshi et. al., in their work turn up with an application "AgroConsultant", an intelligent system that helps in the recommendation of crops based on the different factors with the help of machine learning algorithms (Doshi et al., 2018). They exploit the use of different ML algorithms and finally conclude that the neural networks (NN) technique is far more prudent as it presents higher accuracy compared with other algorithms used. Also, as an enhancement, proposal on rotation of crops as to maximize the yield is made.

As we are predicting different crop yields, one of the predominant crops across the world is Rice. Gandhi and Armstrong in their work use different classification algorithms such as J48, Logical Analysis of Data Tree (LAD Tree), Instance based k-nearest neighbour (IBK), and Locally Weighted Learning (LWL) to compare and predict the yield of rice pertaining to the subtropical climatic zone (7). The factors that they consider affecting the yield of rice are precipitation, minimum temperature, average temperature, maximum temperature, soil type, area in hectares, production in tonnes and historical or past yield in tonnes per hectare. After careful consideration and comparison, they conclude that J48 and LAD tree provides high accuracy, sensitivity and specificity whereas LWL valued the lowest among all the specified criteria. They also specify the importance of having a decision support system for a crop which could work as one stop solution for all the problems.

Likewise, Bhosale et al. (2018) in their work compare different techniques such as K-means clustering, Apriori algorithm and Naïve-Bayes algorithm for the prediction of yield of rice depending on the various parameter with primary focus on Rainfall. With the application of each algorithm, they deduce an appropriate inference that would benefit the farmers either with the prediction of type of crop or approximate crop yield. Ramesh and Vardhan in their work make use of multiple linear regression (MLR) and K-means clustering technique to predict the yield based on rainfall in the Eastern Godavari districts of Andhra Pradesh (Ramesh \& Vardhan, 2013). They claim to have an accuracy of $98 \%$ and $96 \%$ for MLR and K-means technique respectively and also shed light on the importance of $\mathrm{Bi}$-clustering techniques for uncovering the important information related to Agriculture.

Essentially the works that are carried out are intended for the sole purpose of helping farmers and thereby lifting the economy of the country that is very much dependent on Agriculture. Ratkal et al. (2016) in their work titled "Farmer's Analytical Assistant", provide a wonderful and responsive user interface that solves most of the problems that are posed by farmers throughout their life. They also make use of some of the key design elements of multiple linear regression (MLR), online ridge 
regression and stochastic gradient descent to predict the yield and price of crops. The results of this were found to be astonishingly close to the actual market value.

Currently the analytic work related to agriculture is on the upper trend thereby solving all the existing problems at a small chunk. With these works in mind, a decision support system is implemented as part of the work which is detailed in the upcoming sections.

\section{SYSTEM ARCHITECTURE}

The system architecture represents the conceptual model organization in such a way that it backs up the reasoning of the behaviour, structure and other views of the system. Figure 1 gives a clear understanding about the overall process carried out in this work.

Data Collection - The data used for the implementation is acquired from an authenticated and reputed source such as from, University of Agriculture Sciences - Gandhi Krishi Vignan Kendra (GKVK) and other online repositories (http://gismaps.in/prod/indiabasemaps/ Karntaka/Karntaka.html).

Figure 1. Proposed system

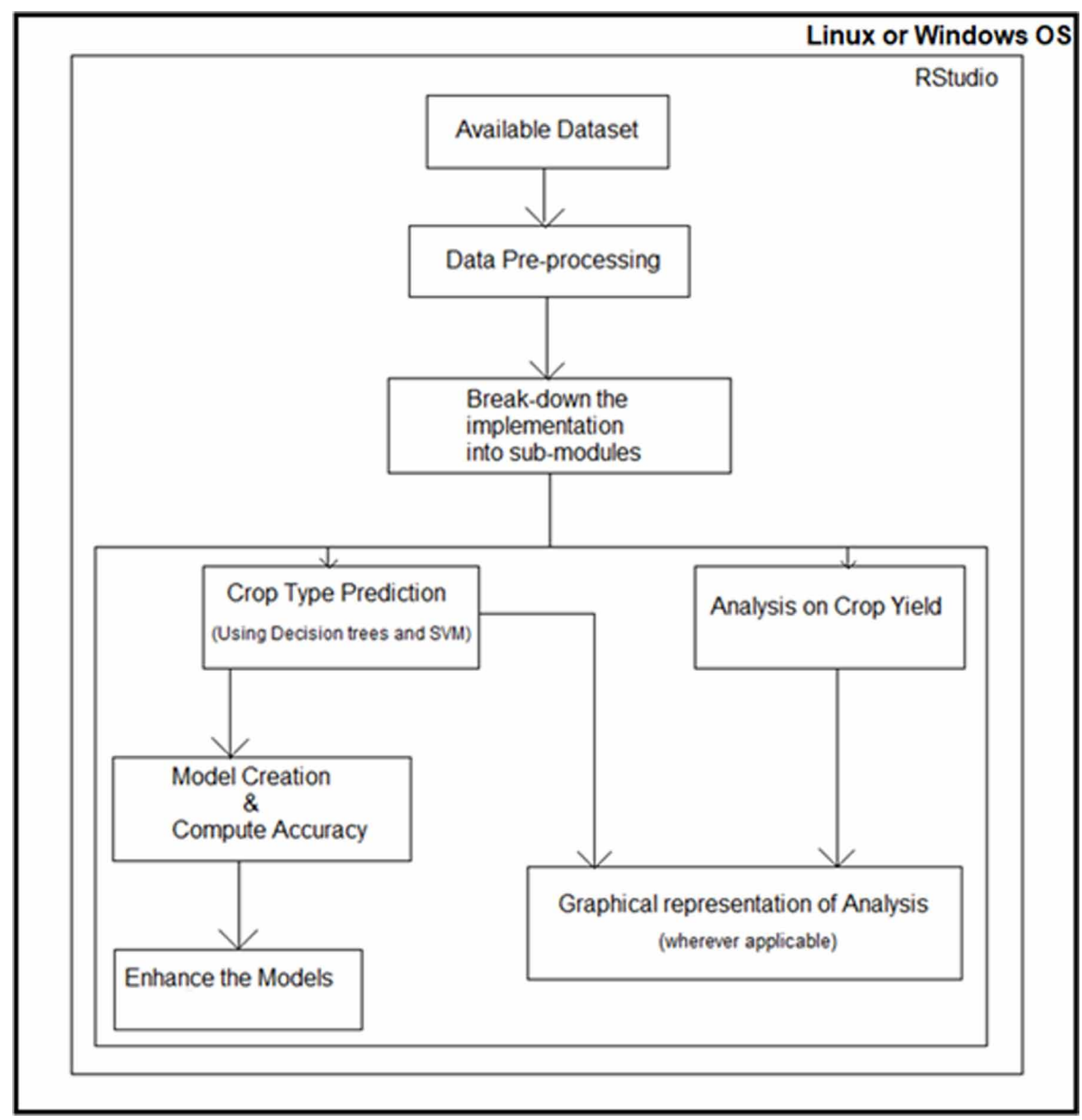


Data Pre-processing - This stage concentrates on the task of pre-processing (transforming the data to the required format) including the handling of missing values.

The implementation is broken down into sub-modules - Crop Type Prediction and Analysis on Crop Yield.

Analysis on Crop Yield - With the focus solely on the state of Karnataka and the major crops grown in the past year, an analysis is accomplished based on the Crop yield and further details pertaining to it are furnished in the implementation and result section.

Crop Type Prediction - This module incorporates the forecast of type of crop, given the agrometeorological data with the help of Decision trees and SVM algorithm. The models are further enhanced by tuning the parameters wherever applicable to obtain a model providing higher accuracy.

Finally, the analysis is picturized with the aid of graphs to provide a clear understanding of the output.

\section{DETAILED EXPLANATION OF THE ANALYTICAL TECHNIQUES USED}

In this work, an attempt is made to predict the type of crop given the agrometeorological data. As the input data is labelled and the output variable (Crop type) is categorical, the Classification algorithms looks to be better suited. Here, two well-known classification algorithms - decision trees and support vector machines (SVM) are used for the implementation. These algorithms are discussed in detail in the following sections.

\subsection{Decision Trees}

A Decision tree is one of the most popular and powerful technique that assists in decision making by utilizing a tree-like model or graph (flow-chart like structure). The internal nodes describe a test (function) on an attribute and each splitting illustrates the consequence or an outcome of the function. The leaf nodes depict the appropriate decision made based on the attributes and the function used for computation. The path from root node to the leaves delineates different classification rules applied for the appropriate outcome (https://medium.com/greyatom/decision-trees-a-simple-way-to-visualizea-decision-dc506a403aeb).

There are different categories of decision tree algorithms such as ID3 (Iterative Dichotomiser), C4.5 (a successor to ID3) and Classification and Regression Trees (CART). Although all the three algorithms follow a greedy approach in the construction of decision trees in a top-down recursive divide-and-conquer fashion, each has its own measure for selection of attributes for splitting the tree.

ID3 uses Information Gain that chooses the split attribute which minimizes the information required to classify the data and reflects the least randomness in the data. But this measure is biased towards the data with many outcomes. The successor of ID3, C4.5 decision tree algorithm uses Gain ratio as the measure to select the splitting attribute which is an addendum to information gain.

As the implementation here is carried out using CART algorithm, the information related to it are discussed in detail. CART uses Gini Index as the measure for selecting splitting attribute of a decision tree. Gini index considers a binary split for each of the attribute. The equation to calculate Gini index is given in Equation (1):

$\operatorname{Gini}(\mathrm{D})=1-\sum_{i=1}^{m} P_{i}^{2}$

Here, $\mathrm{P}_{\mathrm{i}}$ represents the probability that a row in data $\mathrm{D}$ belongs to Class $\mathrm{C}_{\mathrm{i}}$ and is calculated using Equation (2) and the sum is computed over m classes: 


$$
C_{i}=\frac{\left|C_{i, D}\right|}{|D|}
$$

For a binary split, the Gini index is computed as a weighted sum of the impurity of each partition as given in Equation (3):

$\operatorname{Gini}_{\mathrm{A}}(\mathrm{D})=\frac{\left|D_{1}\right|}{|D|} \operatorname{Gini}\left(D_{1}\right)+\frac{\left|D_{2}\right|}{|D|} \operatorname{Gini}\left(D_{2}\right)$

wherein the binary split on an attribute ' $A$ ' partitions the data $\mathrm{D}$ into $\mathrm{D}_{1}$ and $\mathrm{D}_{2}$. The reduction in impurity that would be incurred by a binary split on an attribute A is calculated as in Equation (4):

$\Delta \operatorname{Gini}(\mathrm{A})=\operatorname{Gini}(\mathrm{D})-\operatorname{Gini}_{\mathrm{A}}(\mathrm{D})$

The attribute that maximizes the reduction in impurity is chosen as the splitting attribute and along with its splitting-subset, would together form the splitting criterion (https://machinelearningmastery. com/classification-and-regression-trees-for-machine-learning).

Pruning a decision tree refers to removal of nodes that minimizes the final tree, does not overfit the training data and generalize poorly to new test data without reducing predictive accuracy as computed using the cross-validation set (https://medium.com/@rishabhjain_22692/decision-treesit-begins-here-93ff54ef134).

\subsection{Support Vector Machine (SVM)}

Support vector machine (SVM) is suitable for both regression and classification problems, but is widely used in regards to classification. The goal here is to determine a hyperplane in an $\mathrm{N}$-dimensional space (given the data has $\mathrm{N}$ features), that precisely classifies the data. Hyperplanes are decision boundaries and their dimensions depend upon the number of features in the data (https://towardsdatascience.com/ support-vector-machine-introduction-to-machine-learning-algorithms-934a444fca47).

SVM consists of support vectors, which are the data points that lie closer to the hyperplane and influence the location and direction of the plane and deleting these data points (support vectors) would alter the position of the hyperplane. The simplest way of separating to two groups of data is with a straight line (1 dimension), flat plane (2 dimensions) or an $\mathrm{N}$-dimensional hyperplane (for $\mathrm{N}$ features). An ideal separation is accomplished by the hyperplane which has the maximum distance to the nearest support vector of any class, thereby emphasizing that larger the margin (gap between the hyperplane and support vectors), lower the generalization error of the model (https://en.wikipedia. org/wiki/Support-vector_machine).

Before understanding about the working of SVM, one should be aware of multiclass SVM as it is not the case in daily life to classify into two classes each time. The multiclass SVM aspires to designate labels to instances by using support vector machines, where the labels are selected from a finite set of several elements. SVMs are generally two-class classifiers, meaning they can deduce the data to be belonging to one class or another. The classic way of carrying out multiclass classification is to use one-versus-all (OVA classification) approach, wherein it involves the process of building binary classifiers which distinguish between one of the labels and the rest. Another strategy with multiclass SVM is to construct a set of one-versus-one classifiers and then pick out the class that is chosen from most of the classifiers. The first strategy involves building $\mathrm{ICl}$ number of classifiers, wherein $\mathrm{C}$ corresponds to the number of classes. The second strategy 
requires building $\mid \mathrm{Cl} *(\mid \mathrm{Cl}-1) / 2$ classifiers but the time required for training the classifiers might be less as the training data corresponding to each classifier is significantly less (https://nlp.stanford. edu/IR-book/html/htmledition/multiclass-svms-1.html).

A classic SVM explores to find a margin that isolates all positive and negative examples. However, this may lead to poorly fit models if any examples in the dataset are mislabelled or extremely unusual. There are also some situations, wherein a nonlinear region can segregate the groups more effectively. As far as the data that has been used in this work is non-linear. So, the concept of non-linear SVM is better suited in this case.

SVM handles the grouping of non-linear regions by using a kernel function (related to nonlinear) to portray the data into a distinct space where a hyperplane (linear) cannot be employed to do the segregation. Thereby, with the use of kernel function, a non-linear function is learned by a linear learning machine in a high-dimensional feature space even though the capability of the system is governed by a parameter that is independent to the dimensionality of the space which is called kernel trick.

The SVM linear classifier is expressed as a dot product between data point vectors as in Equation (5). A kernel function must be continuous, symmetric and have a positive definite gram matrix. The most commonly used Kernel function is the Gaussian Radial Basis Function (RBF) which is tantamount to mapping the data into an infinite dimensional Hilbert space, given by the Equation (6):

$\mathrm{K}\left(\vec{x}_{i}, \vec{x}_{j}\right)=\vec{x}_{i}^{T} \cdot \vec{x}_{j}$

$\mathrm{k}\left(\mathrm{x}_{\mathrm{i}}, \mathrm{x}_{\mathrm{j}}\right)=\exp \left(-\gamma\left\|\mathrm{x}_{\mathrm{i}}-\mathrm{x}_{\mathrm{j}}\right\|^{2}\right)$, where $\gamma>0$

where $\mathrm{k}\left(\mathrm{x}_{\mathrm{i}}, \mathrm{x}_{\mathrm{j}}\right)$ is the kernel function, $\mathrm{x}_{\mathrm{i}}$ and $\mathrm{x}_{\mathrm{j}}$ are the data vectors and $\gamma$ defines how far the influence of a single training example reaches. A radial basis function may contain features that take circles (hyperspheres) as hyperplanes, but the decision boundaries turn much more complex if there are interactions of multiple such features (https://nlp.stanford.edu/IR-book/ $\mathrm{html} / \mathrm{htmledition/nonlinear-svms-1.html).}$

\subsection{Enhanced SVM}

The parameters Cost $(\mathrm{C})$ and Gamma $(\gamma)$ are the criteria for an RBF that can be tuned to enhance the existing SVM model to attain more accuracy. Here, the cost $\mathrm{C}$ manages the trade-off between the accurate classification of training points and a smooth boundary. Learning algorithms are mainly focussed on understanding or learning from input data and are not concerned with the means of learning although different means have variety of impact. Due to the bane of dimensionality, the training data pertaining to multi-dimension (usually large number of dimensions) can often be interpreted clearly by over fitting the model. Therefore, generally it is preferable to explicitly concede some training points to be misclassified in order to have an "overall better" position of the separating hyperplane.

If the value of cost is high, then the model chooses more data points as a support vector and therefore the possibility of getting higher variance and lower bias, leading to the problem of overfitting. Conversely, if the value of cost is low, then the model chooses fewer data points as a support vector and thereby obtaining lower variance and high bias leading to underfit model. So, the objective is to find the balance between "not too strict" and "not too loose" value of the cost. Cross-validation and resampling, along with grid search, are some of the efficient ways to procure the best value for cost (https://www.quora.com/What-are-C-and-gamma-with-regards-to-a-support-vector-machine).

As said earlier, the gamma $(\gamma)$ parameter defines how far the influence of a single training example reaches, with low values referring to "far" and high values referring to "close". It 
can be recognized as the inverse of the radius of influence of samples selected by the model as support vectors. If the value of gamma is high, then the decision boundary of the model will depend on data points close to the decision boundary and the closer data points carry more weights than far away points, due to which the decision boundary becomes wigglier. If the value of gamma is low, then far away data points carry more weights than the closer data points and thus the decision boundary looks more like a straight line (https://www. quora.com/What-is-the-intuition-behind-the-Cost-and-Gamma-parameters-in-SVM/answer/ Vibhu-Singh-20).

\section{IMPLEMENTATION}

The implementation of the system is carried out with the help of R programming language and RStudio assists in the implementation. The $\mathrm{R}$ programming language is chosen because of its vast well-defined libraries that supports the data mining algorithms and is also widely used for data analysis.

Some of the important library packages of $\mathrm{R}$ that are needed to implement the analytical algorithms discussed in the previous section are listed below:

- caret: Short for Classification and Regression Training, is one of the modelling functions in R that makes an effort to conduct the process of creation of predictive models (https://cran.r-project. org/web/packages/caret/vignettes/caret.html);

- rpart: Short for Recursive Partitioning and Regression Trees, for building classification and regression models similar to the caret package but has little added functionalities required for recursive calls (https://cran.r-project.org/web/packages/rpart/index.html);

- $\quad$ 1071: A package for miscellaneous functions of the Department of Statistics, Probability and theory group such as for computing Support Vector Machines (SVM), Fourier transforms, naïve-Bayes classifier and many more (https://cran.r-project.org/web/packages/ e1071/index.html).

This work comprises of implementation of 2 modules:

1. Prediction of Crop type:

a. Using Decision trees;

b. Using Support Vector Machines;

2. Analysis on the Yield of the crops:

\subsection{Prediction of Crop Type}

The Prediction of Crop given its agrometeorological data such as Season, Humidity, Temperature and Rainfall, is carried out with the use of two different analytical techniques.

\subsubsection{Use of Decision Trees for the Prediction of Crops}

Here, the models are created based on the decision tree algorithm using the training dataset and the obtained model is cross-validated with the test data to compute the accuracy. The detailed sequence of steps carried out for the implementation are illustrated as shown in Algorithm 1. The decision tree is implemented by calling appropriate functions (rpart) with valid input data, that itself computes and creates the required model. It is for this ease of implementation; $\mathrm{R}$ is preferred as the programming language for this work. The Confusion Matrix is a function in $\mathrm{R}$ that cross-tabulates the original values with the test output values to provide the accuracy of the model. 
Algorithm 1. Prediction of Crop type using Decision trees

Input: Pre-processed data with appropriate attributes. Output: Decision tree model predicting the crop type given the agrometeorological data.

Steps

1. Consider the pre-processed dataset with appropriate attributes (Season, Rainfall, Humidity, Temperature and Crop) required for the algorithm.

2. Subset the data based on attribute (Season) so as to obtain Kharif-crops data and Rabi-crops data.

3. Split both the data obtained in step 2 as training set and testing set (Preferably, use 70:30 random split).

4. Construct the Decision tree model(s) by giving the training set as input.

5. Plot the model to portray the Decision tree.

6. Predict (Cross-validate) the obtained decision tree model obtained in step 4 by using the testing set.

7. Create a ConfusionMatrix to compute the accuracy of the model.

This algorithm splits only on the rainfall attribute, as it provides clear distinction for different crops, when compared to the values of humidity and temperature. Most of the values in humidity and temperature are relatively close for all the crops and hence Rainfall becomes the dominant splitting criterion. In addition, the output obtained is already pruned and hence no further pruning of the model is required.

\subsubsection{Use of Support Vector Machine for the prediction of Crops}

The models are created based on the SVM technique and the sequence of steps are detailed in Algorithm 2. The SVM technique is briefly illustrated in the preceding section and accordingly the computations are carried out even though a library function is used for implementation. As the data is non-linear, the SVM model can be further tuned based on the values of Cost and Gamma and a tuned model is created that provides higher accuracy when compared to the initial SVM model.

While discussing the SVM algorithm, there was also a mention with regards to enhancing the obtained SVM model by fine tuning the parameters $C$ and $\gamma$ using cross validation and grid search. Here, we explicate more on it. Initially, the values of $\mathrm{C}$ and $\gamma$ are unknown and the aim is to determine good values so that the model accurately predicts on the testing data.

Cross-validation is the aspect of splitting the data into ' $x$ ' number of equal sized subsets and sequentially testing one subset using the classifier that is trained with remaining ' $\mathrm{x}-1$ ' subsets. Computing $\mathrm{C}$ and $\gamma$ using cross-validation with "grid-search" incorporates trying out various pairs of values of $(\mathrm{C}, \gamma)$ and selecting a pair that achieves best cross-validation accuracy.

Algorithm 2. Prediction of Crop type using Support Vector Machines (SVM)

Input: Pre-processed data with appropriate attributes. Output: SVM model predicting the Crop type given the agrometeorological data.

Steps

1. Consider the pre-processed dataset with appropriate attributes (Season, Rainfall, Humidity, Temperature and Crop) required for the algorithm. 
2. Subset the data based on attribute (Season) so as to obtain Kharif-crops data and Rabi-crops data.

3. Split both the data obtained in step 2 as training set and testing set (Preferably, use 70:30 random split).

4. Construct the SVM model(s) by providing the training set as input. As the data is non-linear, the obtained model is also Non-Linear.

5. Predict (Cross-validate) the obtained SVM model by using the testing set.

6. Create a ConfusionMatrix to compute the accuracy of the model.

7. Enhance the SVM model by tuning the appropriate values for Cost and Gamma (as detailed in Enhanced SVM).

8. Cross-validate the tuned SVM model(s) and compute the accuracy.

9. Tabulate the accuracy and observe the changes with the appropriate models.

In this work, simple grid search technique is used to compute the values based on two reasons. One, to refrain from performing exhaustive parameter search by heuristics and the other is the computational time is very less when compared to other advanced iterative methods such as walking along a path. Even performing a complete grid-search is time consuming, hence the preferred method is coarse grid first, wherein initially a "better" region on the grid is determined and further, finer grid search in that region is conducted. After finding the best $(\mathrm{C}, \gamma)$ pair, a new model is obtained by using the training set to develop ultimate classifier. The coarse grid first approach works fine for data containing more than thousands of data points and it is appropriate to randomly select a subset of data and perform grid-search on them, rather than on the overall dataset (https://www.csie.ntu. edu.tw/ cjlin/papers/guide/guide.pdf).

\subsection{Analysis on the Yield of the Crops}

The data collected also contains the Area, Production and Yield of different crops across different districts of the country for the last 5 years. By limiting our focus on the districts of Karnataka state, some of the analysis is accomplished and are visually represented by different graphs. The sequence of steps performed for the same are illustrated in Algorithm 3 and the plots are depicted as part of the results section.

Algorithm 3. Sequence of steps for analysis on the crop yield

Input: Data containing the Yield property for different crops Output: Visual representation of the analysis

Steps:

1. Consider the pre-processed data with the required attributes.

2. Subset the data limiting the focus only to the state of Karnataka.

3. Determine the major (at least 5) crops that are cultivated in the past 5 years.

4. Plot the characteristics (Crop vs. Yield) and observe the distribution.

5. As rice, ragi and maize are the 3 major crops grown in Karnataka, with the help of shapefiles data, plot the spread of yield of each crop based on the season (Kharif and Rabi). 


\section{RESULTS}

The Decision tree output for Kharif crops and Rabi crops are depicted in Figure 2 and Figure 3 , respectively. As we can observe from the output, the decision tree algorithm tries to classify the crops only based on the Rainfall attribute, even though all the other parameters are supplied. The left side (path) of the decision tree corresponds to the yes/true value to the condition at each node. Briefly explaining it with reference to Figure 2, sugarcane requires rainfall of more than $200 \mathrm{cms}$, whereas rainfall between $99.5 \mathrm{cms}-200 \mathrm{cms}$ would be better suited for rice. Millet suffices with a rainfall of less than $37.5 \mathrm{cms}$, but Black gram needs rainfall in the margin of $37.5 \mathrm{cms}-57.5 \mathrm{cms}$. Groundnut necessitates rainfall between $80.5 \mathrm{cms}-99.5 \mathrm{cms}$, whereas Green gram and Soybean desires rainfall in the range of $75.5 \mathrm{cms}$ $-80.5 \mathrm{cms}$ and $59.5 \mathrm{cms}-75.5 \mathrm{cms}$.

As we have split the Data into 70:30 train-test split, the cross validation shows that the accuracy of prediction of Kharif and Rabi Crops are $72.04 \%$ and $77.17 \%$, respectively. In addendum, with the tree not considering other parameters further pruning of the tree is not possible. The dataset collected is non-linear, all the factors affect the type of crop, and hence, decision tree model obtained may not be a great judge for the prediction even though it has high accuracy.

The SVM model considers all the factors associated with the data for the prediction of crops. Hence, the SVM plot cannot be possible to implement. But the accuracy of the model is computed by cross validating with the test data and it is found to be $67.72 \%$ and $70.32 \%$ for Kharif and Rabi crops, respectively.

Further the SVM model is tuned to increase the accuracy of prediction by adjusting the values of Cost and Gamma which are necessary values for the non-linear SVM (RBF kernel) classification.

The accuracy of the model after tuning is found to be $68.96 \%$ and $73.23 \%$ respectively for Kharif and Rabi crops. Table 1 shows the accuracy of SVM models before and after tuning. Although, there is no considerable increase in the accuracy, there is slight increase which informs that the tuned model is well-suited for prediction.

The analysis of the crop yield is implemented and the plots obtained are depicted in the following pages.

As we are limiting the focus to Karnataka, the major crops cultivated in different districts of Karnataka for the last 5 years are noted and a plot for the same is depicted as in Figure 4.

From Figure 4, it can be noted that Groundnut, Jowar, Maize, Ragi and Rice are the major crops that are grown in the last 5 years and further analysis on the 3 major crops (Rice, Ragi and Maize) are carried out further.

Figure 2. Decision Tree classification for Kharif crops

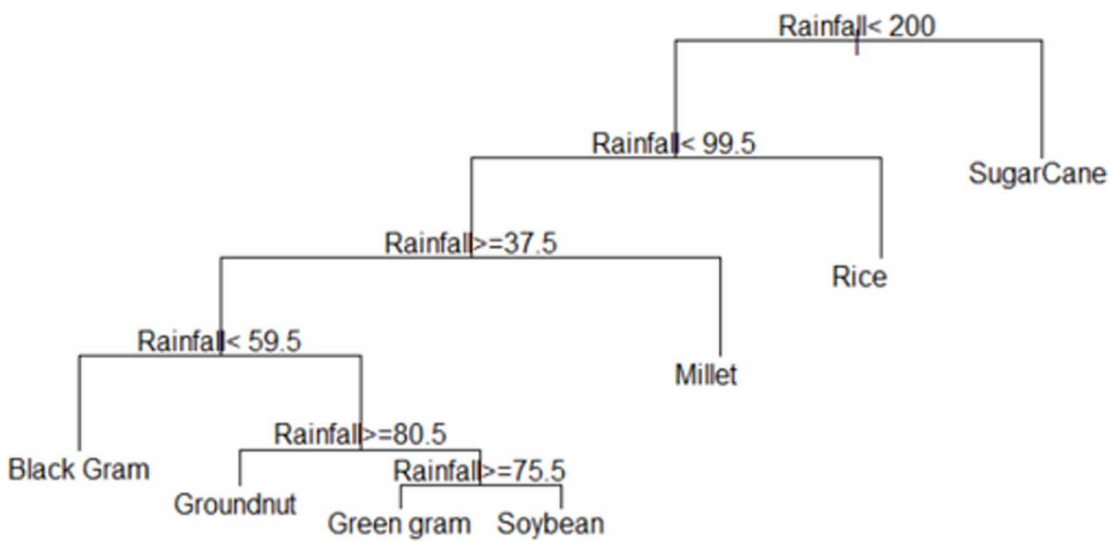




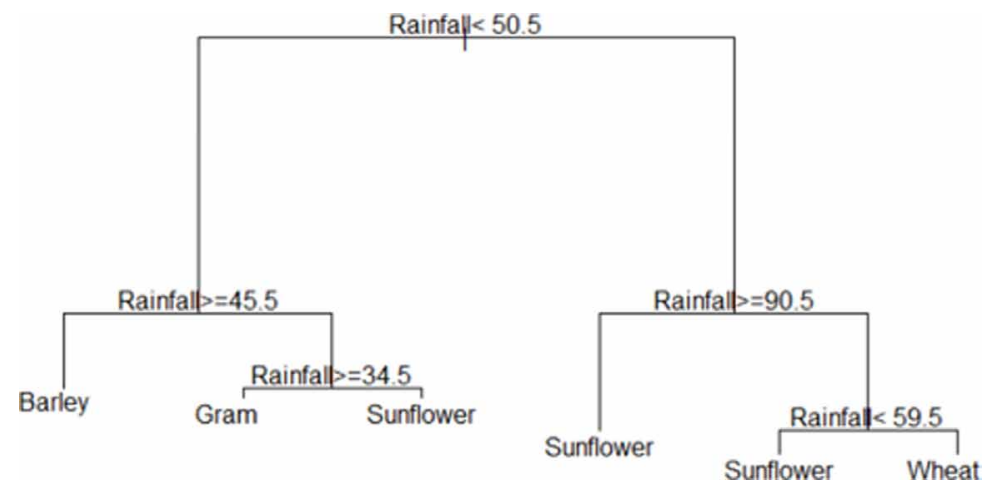

Table 1. Accuracy of SVM models for the crops

\begin{tabular}{|l|l|l|}
\hline & \multicolumn{1}{|c|}{ Kharif Crops } & \multicolumn{1}{c|}{ Rabi Crops } \\
\hline SVM Model (initial) & $67.72 \%$ & $70.32 \%$ \\
\hline SVM Model (after Tuning) & $68.96 \%$ & $73.23 \%$ \\
\hline
\end{tabular}

Figure 4. Boxplot of yield of major crops in Karnataka

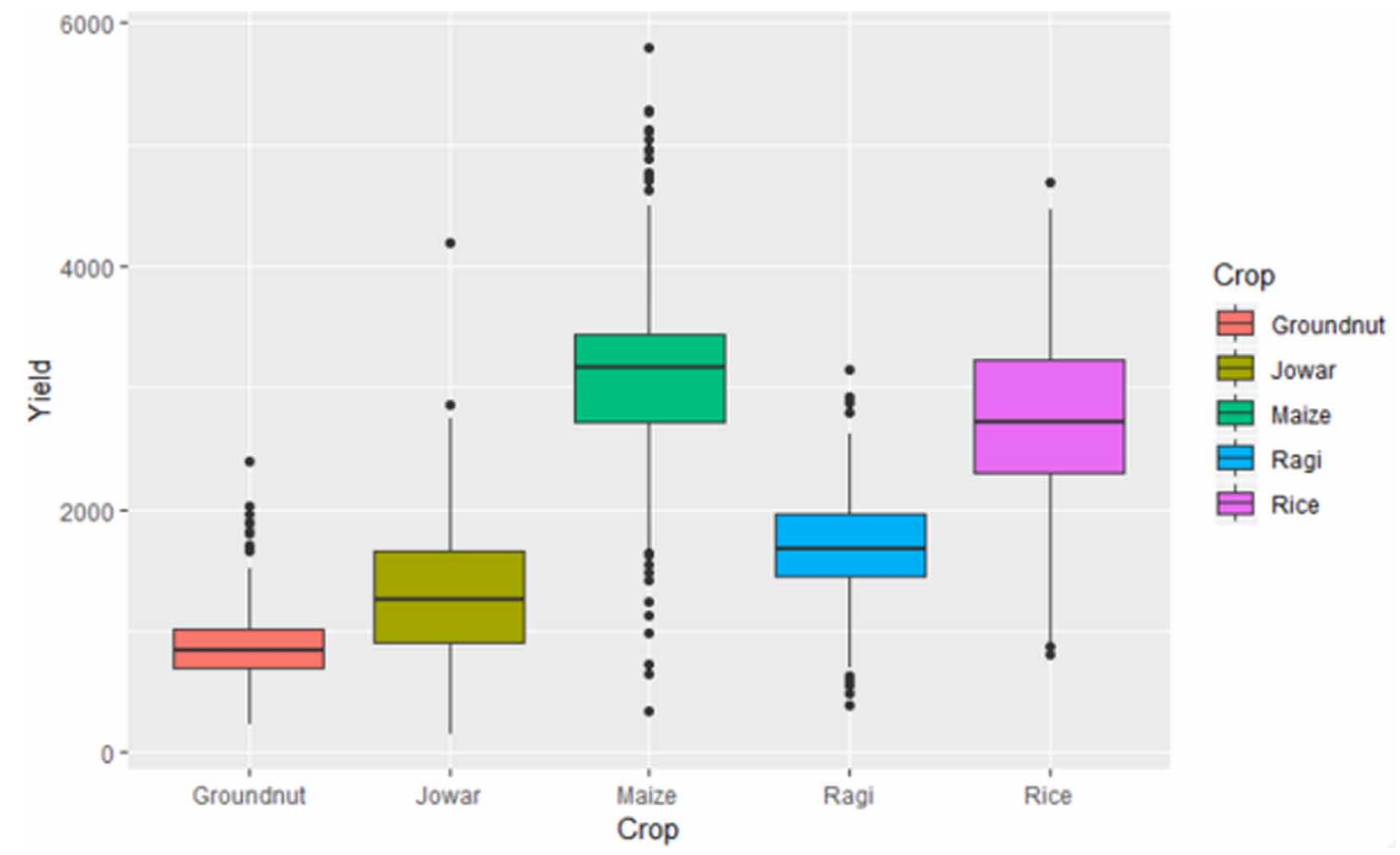

Figure 5 shows the spread of yield of rice across the state of Karnataka during the different season (Kharif and Rabi) for the year 2017. As expected, with Rabi being equivalent to winter the yield of Rice across the state is less when compared to Kharif (autumn) season.

Figure 6 depicts the spread of yield of Ragi across the state for Kharif and Rabi season of the year 2017. By this, it can be observed that the growth of Ragi is mostly cultivated in the middle 
International Journal of Cognitive Informatics and Natural Intelligence

Volume 14 • Issue 2 • April-June 2020

Figure 5. Spread of yield of rice across Karnataka during different season

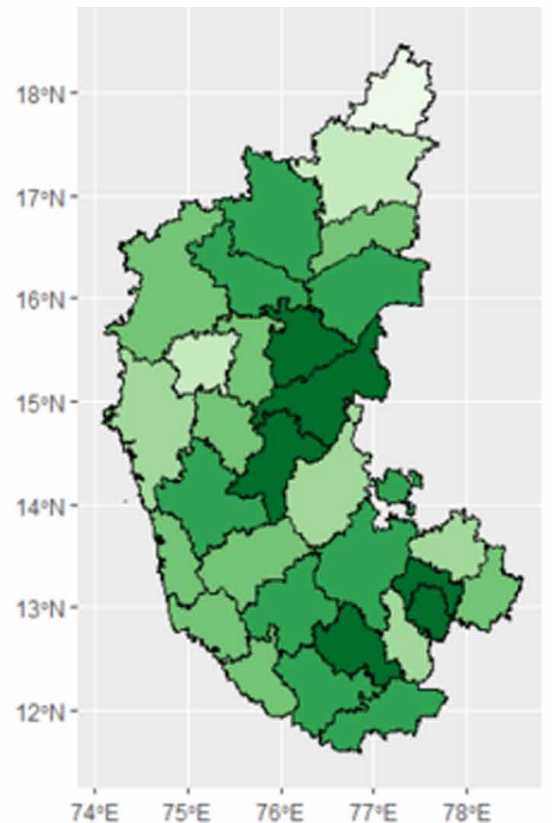

(a)

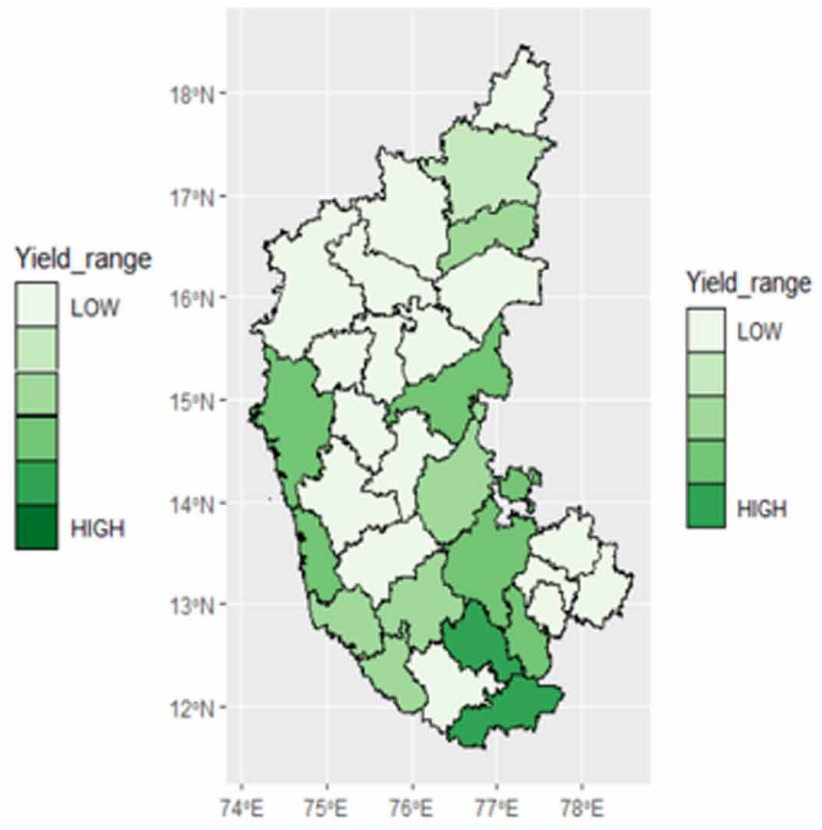

(b) Rabi Season

Figure 6. Spread of Yield of Ragi across Karnataka during different season

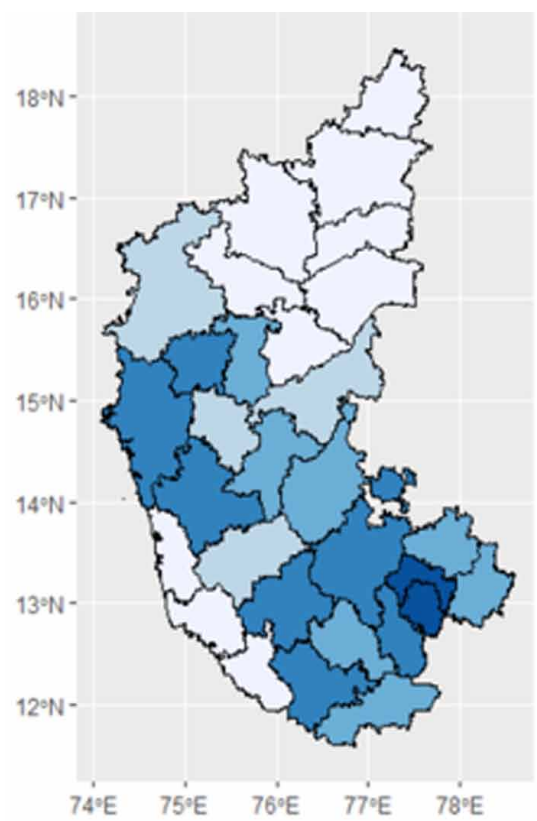

(a) Kharif Season

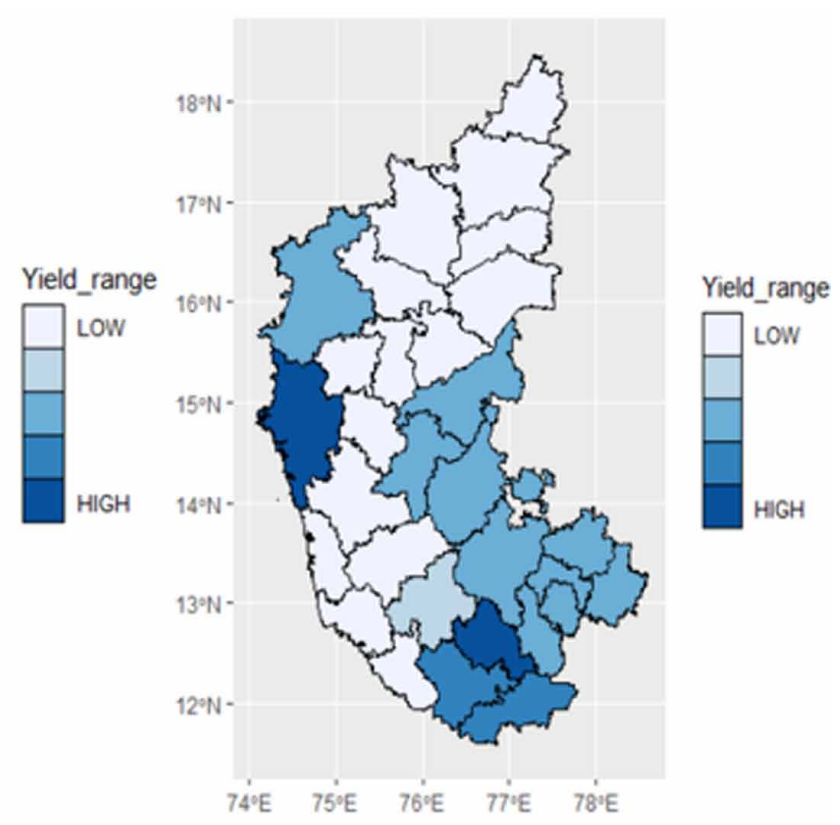

(b) Rabi Season 
and southern districts of the state. There appears to be a dip in the yield during the Rabi season for Ragi as well.

Figure 7 illustrates the spread of Yield of Maize crop across the state of Karnataka. There appears to be uniform distribution of yield for both the seasons. But the yield appears to be slightly more during the Rabi season.

\section{CONCLUSION}

The rapid innovations in tools and technologies in the recent past have driven the field of IoT and data analytics to a great success such that they have been incorporated in all the sectors such as Education, Finance, and Agriculture and have become a part of our daily life. Agriculture is one of the most important sectors especially in a country like India. But in the last few years, agriculture has not contributed as constructively compared to the yesteryears. The reasons for it may be many as listed in the above chapters. So, the use of IoT and data analytics in agriculture is aiding it to move in the right direction. Many authors have analyzed the agriculture data in different ways and have used different techniques and algorithms to help the society. These all are captured as part of the related work in the field.

In this work, an attempt is made to build a decision support system that helps the farmers to make correct decisions, given the appropriate data. The data collected from GKVK consists of agrometeorological information. So, the prediction of type of crop given the information is accomplished by exploiting the classification algorithms Decision trees and Support Vector Machines. The models for both the algorithms are created and accuracy is computed by cross-validation approach. The models are enhanced if possible, by tweaking the appropriate input values that increases the accuracy. As it can be seen that although decision trees provide more accuracy compared to SVM,

Figure 7. Spread of Yield of Maize across Karnataka during different season

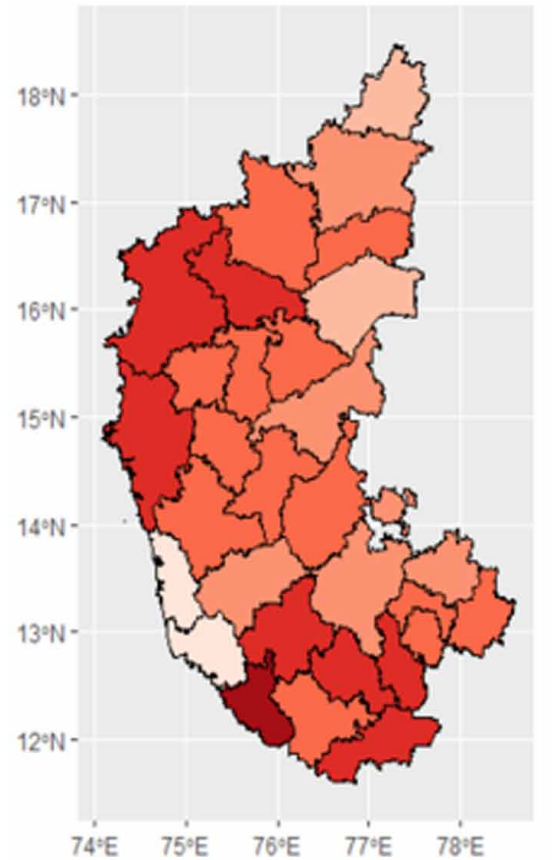

(a) Kharif Season

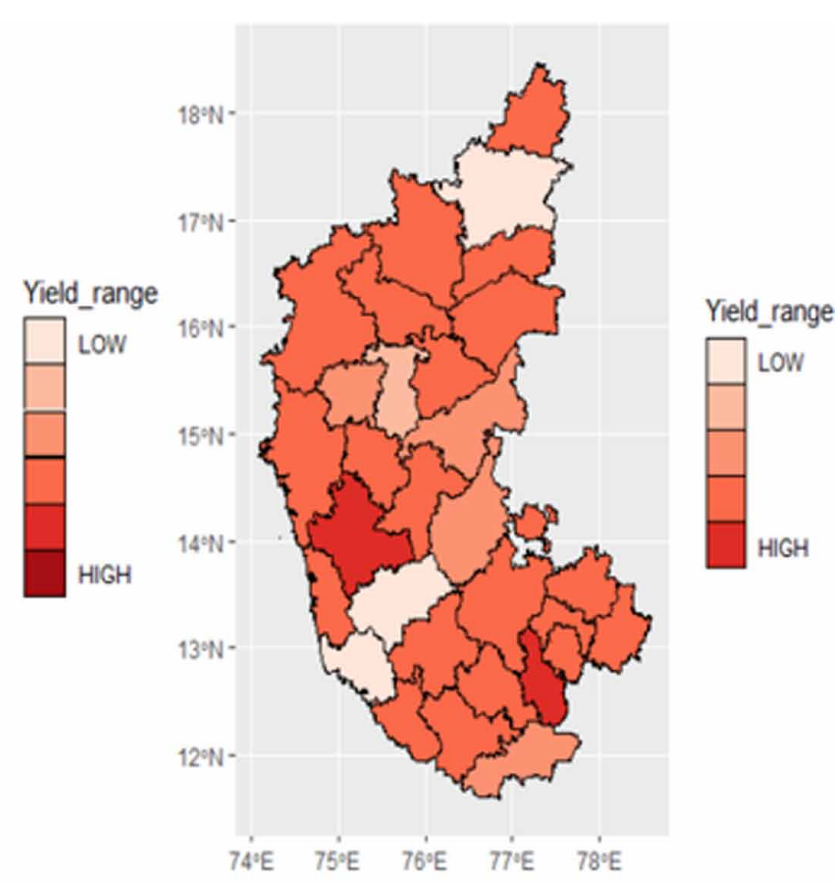

(b) Rabi Season 
but they do not split on all attributes as only Rainfall is considered. But SVM examines all the factors for computation and furnishes a best suited model for the input data.

Also, focusing on the state of Karnataka, the major crops grown across the state in the last five years is calculated and a visual representation for the same is chalked out. With Rice, Ragi and Maize being the top three competitors, the spread of yield of them across the state during different seasons is portrayed.

The future enhancement of any system or application is inevitable due to the explorations occurring all over the world and providing new insights every day. There are many works related to rainfall and water controlling systems that can be incorporated with this system. As the data collected in this case was not feasible to incorporate these, it was not implemented. But with the correct data, many if not all the agriculture work can be embodied into a single system so that it could be very helpful to the farmers. They can have one short stop to put forth their queries and attain invaluable information from the system. Also, with the packages available in R, a web application can be constructed wherein the shiny package aids a lot. 


\section{REFERENCES}

Afrin, S., Khan, A. T., Mahia, M., Ahsan, R., Mishal, M. R., Ahmed, W., \& Rahman, R. M. (2018, June). Analysis of soil properties and climatic data to predict crop yields and cluster different agricultural regions of Bangladesh. Proceedings of the 2018 IEEE/ACIS 17th International Conference on Computer and Information Science (ICIS) (pp. 80-85). IEEE. doi:10.1109/ICIS.2018.8466397

Bhosale, S. V., Thombare, R. A., Dhemey, P. G., \& Chaudhari, A. N. (2018). Crop Yield Prediction Using Data Analytics and Hybrid Approach. Proceedings of the 2018 Fourth International Conference on Computing Communication Control and Automation (ICCUBEA), Pune, India (pp. 1-5). Academic Press. doi:10.1109/ ICCUBEA.2018.8697806

Doshi, Z., Nadkarni, S., Agrawal, R., \& Shah, N. (2018). AgroConsultant: Intelligent Crop Recommendation System Using Machine Learning Algorithms. Proceedings of the 2018 Fourth International Conference on Computing Communication Control and Automation (ICCUBEA), Pune, India. Academic Press. doi:10.1109/ ICCUBEA.2018.8697349

Elijah, O., Rahman, T. A., Orikumhi, I., Leow, C. Y., \& Hindia, M. N. (2018). An Overview of Internet of Things (IoT) and Data Analytics in Agriculture: Benefits and Challenges. IEEE Internet of Things Journal, 5(5), 3758-3773. doi:10.1109/JIOT.2018.2844296

Gandhi, N., \& Armstrong, L. (2016). Applying data mining techniques to predict yield of rice in humid subtropical climatic zone of India. Proceedings of the 2016 3rd International Conference on Computing for Sustainable Global Development (INDIACom), New Delhi (pp. 1901-1906). Academic Press.

Pudumalar, S., Ramanujam, E., Rajashree, R. H., Kavya, C., Kiruthika, T., \& Nisha, J. (2017). Crop recommendation system for precision agriculture. Proceedings of the 2016 Eighth International Conference on Advanced Computing (ICoAC), Chennai, India (pp. 32-36). Academic Press. doi:10.1109/ICoAC.2017.7951740

Ramesh, D., \& Vardhan, B. V. (2013). Data mining techniques and applications to agriculture yield data. International Journal of Advanced Research in Computer and Communication Engineering, 2(9), 3477-3480.

Ratkal, A. G., Akalwadi, G., Patil, V. N., \& Mahesh, K. (2016). Farmer's Analytical Assistant. Proceedings of the 2016 IEEE International Conference on Cloud Computing in Emerging Markets (CCEM), Bangalore (pp. 84-89). IEEE. doi:10.1109/CCEM.2016.023

Sujatha, R., \& Isakki, P. (2016). A study on crop yield forecasting using classification techniques. Proceedings of the 2016 International Conference on Computing Technologies and Intelligent Data Engineering (ICCTIDE'16), Kovilpatti (pp. 1-4). Academic Press. doi:10.1109/ICCTIDE.2016.7725357

Vijayabaskar, P. S., Sreemathi, R., \& Keertanaa, E. (2017). Crop prediction using predictive analytics. Proceedings of the 2017 International Conference on Computation of Power, Energy Information and Communication (ICCPEIC) (pp. 370-373). Academic Press. doi:10.1109/ICCPEIC.2017.8290395 